\title{
INFLUÊNCIA DAS VARIÁVEIS NITROGÊNIO, FÓSFORO E 'BRIX NA PRODUÇÃO DOS METABÓLITOS SECUNDÁRIOS CONTAMINANTES TOTAIS DA FERMENTAÇÃO ALCOÓLICA
}

\section{José Alexsandro Silva*}

Departamento de Farmácia, Universidade Estadual da Paraíba, Rua Juvêncio Arruda, s/n, Campus Universitário, 58109-790 Campina Grande - PB, Brasil

Flávio Luiz Honorato da Silva

Departamento de Engenharia Química, Centro de Ciência e Tecnologia, Universidade Federal de Campina Grande, Av. Aprígio Veloso, 882, 58109-970 Campina Grande - PB, Brasil

\section{Rômulo Romeu da Nóbrega Alves}

Departamento de Biologia, Universidade Estadual da Paraíba, Rua Juvêncio Arruda, s/n, Campus Universitário, 58109-790 Campina Grande - PB, Brasil

Davi Pereira de Santana

Departamento de Ciências Farmacêuticas, Universidade Federal de Pernambuco, 50739-520 Recife - PE, Brasil

Recebido em 13/4/05; aceito em 23/9/05; publicado na web em 12/4/06

\begin{abstract}
INFLUENCE OF NITROGEN, PHOSPHORUS AND ${ }^{\circ}$ BRIX ON THE PRODUCTION OF TOTAL SECONDARY CONTAMINANT METABOLITES (MSCT) OF ALCOHOLIC FERMENTATION. The present study evaluated the influence of nitrogen, phosphorus and ${ }^{\circ}$ Brix on the production of MSCT through a factorial design methodology and analysis of response surface. The objective was to propose a statistically significant probabilistic model for the alcoholic fermentation. In order to obtain less MSCT, the medium under fermentation needs to present low sugar concentration. It was observed that phosphorus and nitrogen, even with no significant individual effects, presented interactions with each other decreasing the production of MSCT, which improves alcohol quality.
\end{abstract}

Keywords: factorial design; ethanol; response surface.

\section{INTRODUÇÃO}

A importância sócio-econômica da exploração da cultura canavieira em solos brasileiros é reconhecida desde os tempos do Brasil colônia. Atualmente, além dos aspectos sócio-econômicos, essa cultura tem sua importância reconhecida também nas áreas econômica e ambiental, tendo o álcool como uma fonte renovável de energia menos poluente, que integra parte da matriz energética nacional e está sendo um dos produtos mais importantes do mercado internacional ${ }^{1}$.

Atualmente, o Brasil posiciona-se no cenário internacional como o maior produtor e exportador mundial de açúcar de cana e o maior produtor e consumidor de álcool produzido por fermentação ${ }^{2}$, sendo também o único país a introduzir, em larga escala, o álcool como combustível alternativo ao petróleo.

A produção de álcool no Brasil é feita quase que exclusivamente por fermentação de mosto, constituído por caldo de cana ou melaço, ou ainda, por misturas destes dois componentes. Alguns macronutrientes e/ou micronutrientes também são adicionados ao mosto, a fim de complementar as deficiências do meio, quanto a certos elementos indispensáveis às leveduras para máxima transformação dos açúcares. O microrganismo agente da fermentação mais utilizado é um fungo leveduriforme, Saccharomyces cerevisiae ${ }^{3}$.

Não existe uma especificação nacional, ou mesmo internacional, que contemple todos os tipos de álcool em comercialização. As especificações solicitadas pelos compradores dependem diretamente do uso específico, ou ainda, se o material adquirido será reprocessado. A denominação álcool neutro procura englobar diversos "tipos" de álcool, por ex., álcool fino, álcool extra-fino, álcool de qualidade industrial. Sendo assim, o álcool neutro é uma denominação do álcool, hidratado ou anidro, que apresenta baixos

*e-mail: alexuepb@yahoo.com.br teores de impurezas e dos metabólitos secundários contaminantes totais - MSCT ${ }^{4}$.

Na produção de álcool, o fosfato apresenta importância na fermentação, principalmente no início das safras, em virtude da necessidade da produção de quantidade apreciável de massa celular ${ }^{5}$. Em relação ao nitrogênio, a forma mais utilizada pela levedura na fermentação é a amoniacal, porém, na sua ausência, a levedura utiliza outras fontes como os aminoácidos, o que proporciona um aumento nos níveis dos álcoois superiores (n-propanol, iso-butanol, n-butanol, iso-amílico) $)^{6,7}$.

Este estudo teve o objetivo de avaliar a influência das variáveis nitrogênio, fósforo e ${ }^{\circ}$ Brix na produção dos MSCT (substâncias orgânicas voláteis - aldeído, acetonas, acetato de etila, metanol; e álcoois superiores - n-propanol, iso-butanol, n-butanol e isoamilico) através da metodologia de planejamento fatorial e análise de superfícies de resposta, visando propor um modelo probabilístico estatisticamente significativo na fermentação alcoólica.

\section{PARTE EXPERIMENTAL}

\section{Caldo de cana (substrato)}

Foi utilizado como substrato o caldo de cana da variedade CB 453 do talhão 2045, que é irrigada com vinhaça e rotineiramente utilizado na Destilaria Japungu, Santa Rita - PB, para produção de álcool.

\section{${ }^{\circ}$ Brix do caldo in natura e do mosto}

Para o doseamento do ${ }^{\circ}$ Brix do caldo in natura, realizado antes das fermentações no laboratório de sacarose da Destilaria Japungu, foi utilizado um refratômetro digital automático da marca Acatec RDA 8600. O ${ }^{\circ}$ Brix do mosto, dosado durante o processo de fer- 
mentação, foi determinado em um sacarímetro de ${ }^{\circ}$ Brix modelo TP 20/C da marca Incoterm.

\section{Determinação dos metabólitos secundários contaminantes totais (MSCT)}

A determinação dos MSCT foi realizada por cromatografia gasosa, utilizando-se um cromatógrafo GC 90, equipado com uma coluna empacotada, nas seguintes condições de operacionais: temperatura de 130,160 e $86{ }^{\circ} \mathrm{C}$ para injeção, detector e coluna, respectivamente. $\mathrm{O}$ nitrogênio foi utilizado como gás de arraste com velocidade de $20 \mathrm{~mL} / 40 \mathrm{~s}$.

Para determinação dos MSCT, $5 \mu \mathrm{L}$ do vinho delevurado ou centrifugado foram injetados diretamente no cromatógrafo, na mesma coluna que analisava o etanol, porém acrescentando-se uma précoluna para evitar sangramentos ou danos à coluna propriamente dita, imediatamente após o final das fermentações. Foi utilizado um padrão, adquirido no ITEP (Instituto Tecnológico de Pernambuco), com as seguintes concentrações: aldeído: 3,15; acetona: 3,02; acetato de etila: 4,96; metanol: 2,73; n-propanol: 6,03; iso-butanol: 4,42; nbutanol: 3,10 e iso-amílico: 3,20 mg/100 mL.

\section{Delineamento experimental}

O caldo de cana utilizado nas fermentações foi diluído na concentração do ${ }^{\circ}$ Brix desejado, conforme o nível do planejamento experimental. O nitrogênio e o fósforo foram adicionados, também, conforme o nível de cada experimento. Após o ajuste das concentrações de ${ }^{\circ}$ Brix, nitrogênio e fósforo, os caldos foram esterilizados em autoclave a $121{ }^{\circ} \mathrm{C}, 1 \mathrm{~atm}$ de pressão por $30 \mathrm{~min}$, sendo posteriormente resfriados à temperatura ambiente.

\section{Processo de fermentação}

Para o processo de fermentação foi utilizada a cepa Saccharomyces cerevisiae IA1234, obtida a partir do fermento (leite puro) proveniente da fermentação alcoólica da Destilaria Japungu. Este material foi recolhido nos pré-fermentadores, que são os tanques onde se realiza o tratamento do fermento a ser utilizado na produção de álcool, minutos antes de se realizar a inoculação no caldo de cana. Para cada fermentação foram adicionados $250 \mathrm{~mL}$ de leite puro (fermento), que corresponde a uma concentração de $10 \%$ v/v do caldo.

As fermentações foram realizadas em balão de fundo chato de $6000 \mathrm{~mL}$, que foram tampados com algodão hidrófilo. O acompanhamento do processo de fermentação foi realizado por medição horária do ${ }^{\circ}$ Brix, considerando-se como o fim da fermentação quando o consumo de açúcares cessava, ou quando o valor do ${ }^{\circ}$ Brix repetia por no mínimo duas vezes.

\section{RESULTADOS E DISCUSSÃO}

\section{Otimização do processo}

O objetivo do uso da metodologia de planejamento fatorial é encontrar um modelo empírico que possa predizer bem o processo em estudo, avaliando a influência das variáveis de entrada, ${ }^{\circ}$ Brix, nitrogênio e fósforo, sobre a variável resposta MSCT (substâncias orgânicas voláteis - aldeído, acetona, acetato de etila, metanol e os álcoois superiores - n-propanol, iso-butanol, n-butanol e iso-amílico).

Estudando-se a influência das variáveis de entrada sobre a variável resposta, foi definida a faixa ótima de operações do processo de fermentação para obtenção de um álcool de melhor qualidade, que é aquela com o mínimo possível de MSCT. A utilização da metodologia de planejamento fatorial em estudos que abrangem muitas variáveis torna possível o planejamento e a realização de maneira organizada de uma quantidade mínima necessária de experimentos ${ }^{8-10}$.

\section{Planejamento experimental}

Para avaliar a influência das variáveis independentes - ${ }^{\circ}$ Brix, nitrogênio e fósforo - sobre a variável dependente MSCT, elaborou-se um planejamento fatorial de 2 níveis $(-1$ e +1$)$ mais configuração estrela, aqui chamado de modelo de $2^{\mathrm{a}}$ ordem, totalizando 16 experimentos laboratoriais, os quais foram executados aleatoriamente e em duplicatas, conforme matriz do planejamento fatorial mostrada na Tabela 1 .

Tabela 1. Matriz do planejamento fatorial $2^{3}+$ configuração estrela com a resposta MSCT $(\mathrm{mg} / 100 \mathrm{~mL})$

\begin{tabular}{cccc}
\hline Nitrogênio & Fósforo & ${ }^{\circ}$ Brix & MSCT \\
\hline-1 & -1 & -1 & 18,7181 \\
+1 & -1 & -1 & 18,6099 \\
-1 & +1 & -1 & 29,9309 \\
+1 & +1 & -1 & 21,3784 \\
-1 & -1 & +1 & 32,3096 \\
+1 & -1 & +1 & 32,9449 \\
-1 & +1 & +1 & 35,0436 \\
+1 & +1 & +1 & 30,3313 \\
$-1,682$ & 0 & 0 & 25,2756 \\
$+1,682$ & 0 & 0 & 26,8977 \\
0 & $-1,682$ & 0 & 24,4970 \\
0 & $+1,682$ & 0 & 25,8282 \\
0 & 0 & $-1,682$ & 18,2750 \\
0 & 0 & $+1,682$ & 33,3172 \\
0 & 0 & 0 & 24,8994 \\
0 & 0 & 0 & 23,5153 \\
\hline
\end{tabular}

* Os valores dos MSCT são referentes à média de dois cromatogramas

Na Tabela 2 estão expressos os valores reais e os níveis dos fatores estudados nos 16 experimentos. As faixas estudadas neste planejamento fatorial foram definidas tendo como base valores comumente utilizados em muitas destilarias de álcool, principalmente na região Nordeste e, em especial, na Destilaria Japungu.

O modelo matemático empírico, codificado de $2^{\mathrm{a}}$ ordem encontrado, com os seus respectivos parâmetros estatísticos e desvios padrão, está apresentado na Equação 1, onde os valores em negrito são os parâmetros estatisticamente significativos. Este modelo foi

Tabela 2. Valores reais e os níveis dos fatores estudados para o planejamento fatorial completo $\left(2^{3}\right)+$ configuração estrela do processo fermentativo

\begin{tabular}{lccccc}
\hline Variáveis & Nível $-1,682$ & Nível-1 & Ponto central & Nível +1 & Nível +1,682 \\
\hline $\mathrm{N}(\mathrm{g} / \mathrm{L})$ & 0,1 & 0,3 & 0,6 & 0,9 & 1,1 \\
$\mathrm{P}(\mathrm{g} / \mathrm{L})$ & 0,002 & 0,05 & 0,12 & 0,19 & 0,24 \\
${ }^{0}$ Brix $(\%)$ & 10 & 12 & 15 & 18 & 20 \\
\hline
\end{tabular}


obtido a partir da regressão não linear (modelo quadrático) dos dados experimentais, utilizando o software Statistica versão 5.0.

MSCT $=(\mathbf{2 4 , 0 5} \pm \mathbf{1 , 6 8})-(0,7328 \mathrm{~N} \pm 0,6360)+(1,1964 \mathrm{P} \pm$ $0,6360)+\left(\mathbf{4 , 9 2 6 9}{ }^{\mathbf{0}} \mathbf{B r i x} \pm \mathbf{0 , 6 3 6 0}\right)+\left(1,0454 \mathrm{~N}^{2} \pm 0,7721\right)+$ $\left(0,7188 \mathrm{P}^{2} \pm 0,7721\right)+\left(0,9427{ }^{\circ} \mathrm{Brix}^{2} \pm 0,7721\right)-(\mathbf{1 , 7 2 4 0} \mathbf{~ N x P} \pm$ $\mathbf{0 , 8 3 1 0})+\left(0,5730 \mathrm{Nx}^{\mathrm{o}} \mathrm{Brix} \pm 0,8310\right)-\left(\mathbf{1 , 7 3 2 6} \mathbf{P x}{ }^{\circ} \mathrm{Brix} \pm\right.$ 0,8310)

Na Tabela 3 estão apresentados os resultados da ANOVA (Análise de Variância) para a resposta MSCT. Verificou-se que o ajuste do modelo foi satisfatório, com coeficiente de correlação de 0,9629 e percentagem de variância explicada de $92,72 \%$. A Figura 1 mostra os valores preditos (modelo empírico, Equação 1) versus os valores experimentais para os MSCT, observando-se uma boa concordância. $\mathrm{O}$ teste $\mathrm{F}$ também mostra que o valor de $\mathrm{F}_{\text {calculado }}=8,49$ é duas vezes maior que o valor de $\mathrm{F}_{\text {tabelado }}=2,96$, com $90 \%$ de confiança, mostrando que o modelo foi bem ajustado, sendo este estatisticamente significativo ${ }^{8}$.

Tabela 3. Análise de Variância para a variável resposta MSCT

\begin{tabular}{lccc}
\hline $\begin{array}{l}\text { \% variância } \\
\text { explicada }\end{array}$ & $\begin{array}{c}\text { Coeficiente } \\
\text { de correlação }\end{array}$ & Teste F & $\begin{array}{c}\text { F tabelado para } \\
90 \% \text { confiança }\end{array}$ \\
\hline 92,72 & 0,9629 & 8,49 & 2,96 \\
\hline
\end{tabular}

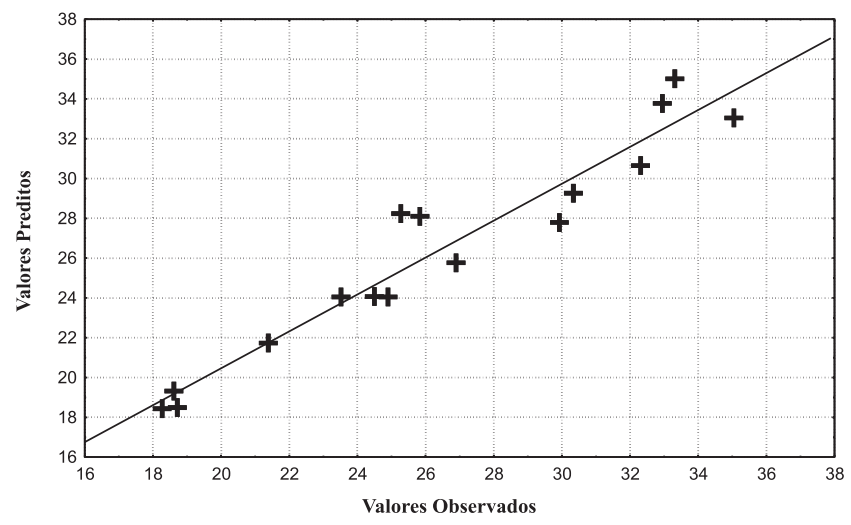

Figura 1. Valores observados versus valores preditos para o MSCT

Considerando que o modelo quadrático foi válido para a variável resposta MSCT, o mesmo foi usado para gerar as superfícies de resposta, tendo o objetivo de otimizar (minimizar a produção de MSCT) o processo.

Objetivando encontrar as faixas das variáveis em estudo que minimizem as concentrações de MSCT no fermentado foi construída a Figura 2, que mostra os efeitos das variáveis concentração de nitrogênio e de fósforo, fixando-se a variável ${ }^{\circ}$ Brix (variável de maior influência) no nível -1 (12\%). Operando-se em concentração de nitrogênio abaixo de $0,6 \mathrm{~g} / \mathrm{L}$ e concentração de fósforo em torno $0,002 \mathrm{~g} / \mathrm{L}$, obteve-se as menores concentrações de MSCT (aproximadamente 15,5 mg/100 mL).

A Figura 3 mostra o efeito do nitrogênio e do ${ }^{\circ}$ Brix sobre a produção dos MSCT, tendo o valor do fósforo fixado no ponto central $(0,12 \mathrm{~g} / \mathrm{L})$. Observa-se que nas fermentações com ${ }^{\circ}$ Brix baixo (11\%) e com a concentração de nitrogênio elevada, entre 0,6 a 1,1 $\mathrm{g} / \mathrm{L}$, obteve-se uma menor concentração dos MSCT.

A Figura 4 apresenta o efeito do fósforo e do ${ }^{\circ}$ Brix sobre a resposta MSCT com nitrogênio fixado no ponto central $(0,6 \mathrm{~g} / \mathrm{L})$, onde se verifica que a menor produção de MSCT ocorreu quando o fósforo está em concentração baixa (abaixo de 0,05 g/L) e o ${ }^{\circ}$ Brix abaixo de $11 \%$.

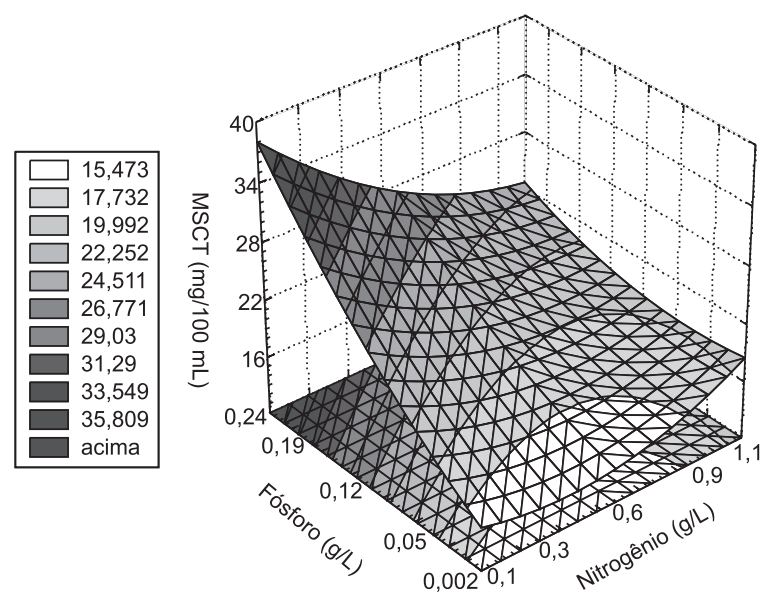

Figura 2. Superfícies de resposta dos efeitos do fósforo e do nitrogênio, com $o^{\circ}$ Brix fixado em $12 \%$ (nível-1) para MSCT

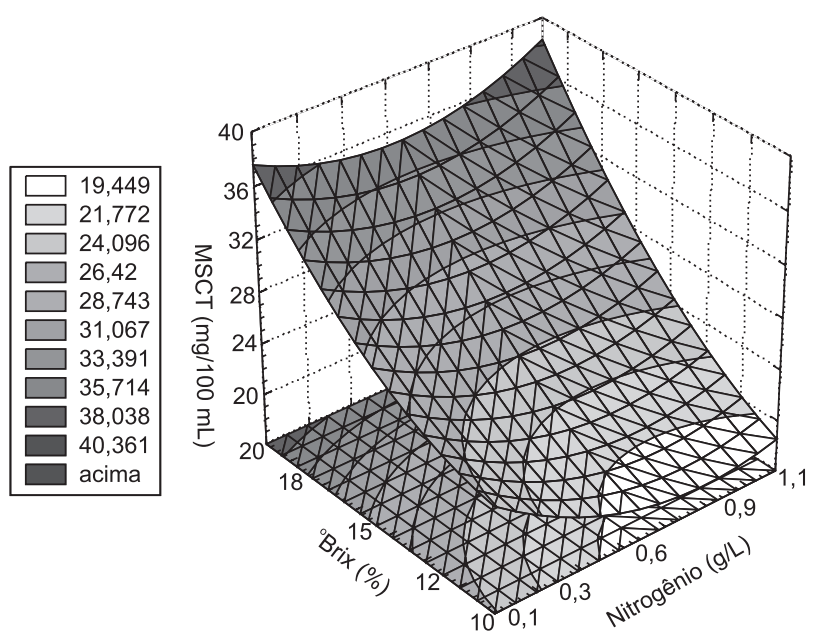

Figura 3. Superfícies de resposta dos efeitos do nitrogênio e do ${ }^{\circ}$ Brix para o fósforo fixado em 0,12 g/L (ponto central) para MSCT

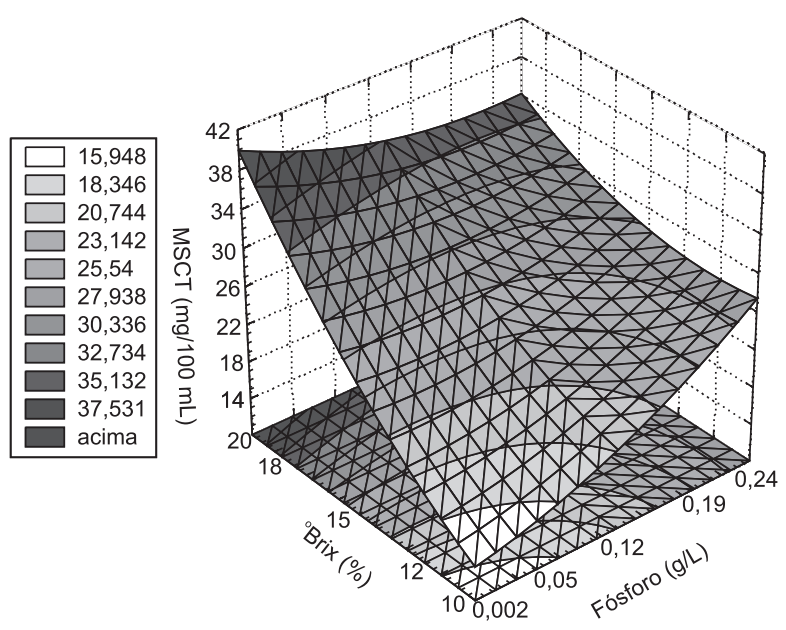

Figura 4. Superfícies de resposta dos efeitos do fósforo e do ${ }^{\circ}$ Brix para o nitrogênio fixado em 0,6 g/L (ponto central) para MSCT 
A Figura 5 apresenta o efeito do nitrogênio e do ${ }^{\circ}$ Brix sobre a resposta MSCT com fósforo fixado no nível inferior -1,682 (0,002 $\mathrm{g} / \mathrm{L}$ ), onde se verifica que a menor produção de MSCT ocorreu quando o ${ }^{\circ}$ Brix está abaixo de $11 \%$ e o nitrogênio em uma faixa ampla de 0,1 a 0,9 g/L. Operando-se a fermentação nestas faixas, obteve-se concentração de MSCT de no máximo 15,8 mg/100 mL.

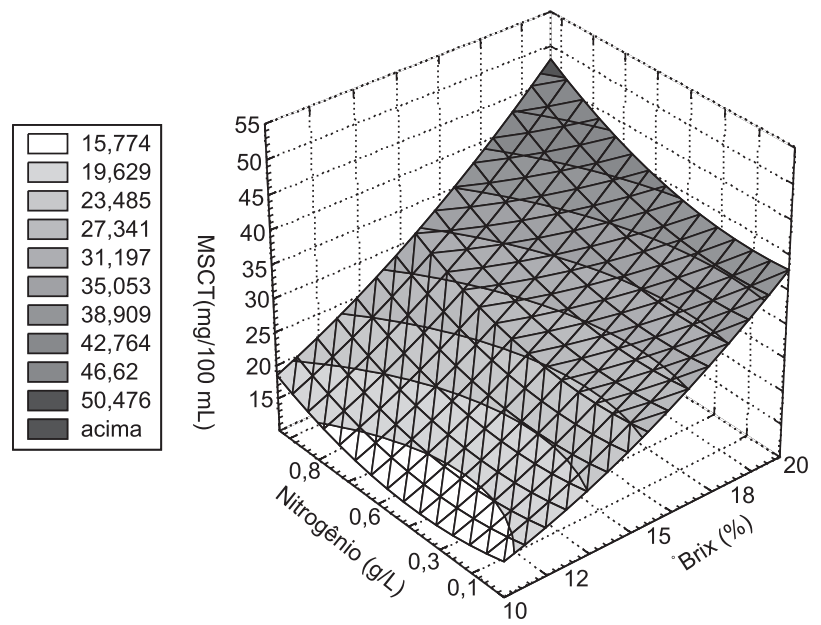

Figura 5. Superfícies de resposta dos efeitos do nitrogênio e do ${ }^{\circ}$ Brix para o fósforo fixado em 0,002 $\mathrm{g} / \mathrm{L}$ (nível -1,682) para MSCT

A Figura 6 apresenta a influência das fontes dos nutrientes fósforo e nitrogênio sobre a formação de MSCT $(\mathrm{mg} / 100 \mathrm{~mL})$, fixando-se o ${ }^{\circ}$ Brix no nível inferior de -1,682 (10\%). Observa-se que operando o processo fermentativo com adição da fonte de fósforo abaixo de $0,05 \mathrm{~g} / \mathrm{L}$, para qualquer valor da fonte de nitrogênio variando de 0,1 a $0,9 \mathrm{~g} / \mathrm{L}$, obteve-se MSCT mínimo de aproximadamente $15,7 \mathrm{mg} / 100 \mathrm{~mL}$.
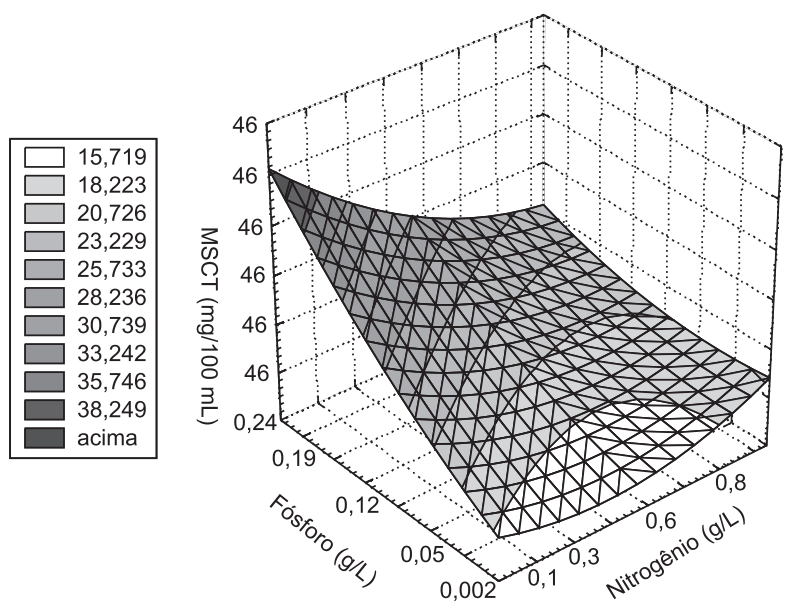

Figura 6. Superfícies de resposta dos efeitos do fósforo e do nitrogênio, com $o^{\circ}$ Brix fixado em $10 \%$ (nível-1,682) para MSCT

Pode-se afirmar que a adição das fontes de fósforo e nitrogênio é importante para o processo de fermentação alcoólica (maior produção de etanol). No caso em estudo (minimização da produção de MSCT), principalmente os álcoois superiores, a adição da fonte de nitrogênio é benéfica. Todavia, evidenciou-se, também, que a fonte de fósforo acelera o aumento da formação de MSCT, como observado nas análises das superfícies de resposta em estudo. Contudo, mesmo não sendo observadas influências individuais (efeitos principais - 1a ordem, Equação 1) destes nutrientes, a interação entre as mesmas (PxN) influencia na resposta MSCT; sendo assim, pode-se concluir que a fonte de nitrogênio tende a influenciar a diminuição da formação de MSCT, enquanto a fonte de fósforo apresenta um efeito de formação dos MSCT.

Foi possível verificar uma relação diretamente proporcional entre a produção de MSCT e a de etanol pois, com o aumento do ${ }^{\circ}$ Brix no meio, há aumento da produção do etanol, sem observar a inibição pelo substrato ${ }^{4}$.

Além de melhorar a qualidade do álcool etílico obtido, a diminuição dos MSCT no meio auxilia a eficiência do processo fermentativo, pois estes apresentam certa toxicidade para a levedura, inibindo-a ${ }^{11}$.

Já se escreveu muito sobre a necessidade ou não de suplementação de nutrientes no caldo de cana, principalmente fósforo e nitrogênio, porém, o assunto continua sendo discutido e questiona$\mathrm{do}^{4}$, pois uns concluem que a adição é benéfica e outros questionam o tema, não encontrando efeitos significativos em tal suplementação $0^{3,5-7,12-14}$.

Neste trabalho o que se pode afirmar é que a adição destes nutrientes, principalmente o nitrogênio, na resposta MSCT é benéfica para a redução da produção de MSCT, até certo limite, como comprovado e observado nas superfícies de resposta. Já em relação ao fósforo, o mesmo deve estar em baixas concentrações no meio fermentativo (substrato), principalmente quando se deseja produzir álcool etílico hidratado ou anidro extrafino, que é o interesse das indústrias alcoolquímicas, farmacêuticas e de bebidas.

\section{AGRADECIMENTOS}

À Destilaria Japungu - Santa Rita-PB pelo suporte financeiro do Projeto e ao CNPq pela concessão de bolsa de mestrado.

\section{REFERÊNCIAS}

1. Ros, P. B.; Dissertação de Mestrado, Universidade de São Paulo, Brasil, 2004.

2. Saito, J. R.; Dissertação de Mestrado, Universidade Federal de São Carlos, Brasil, 2000.

3. Stupiello, J. P.; Horii, J.; STAB 1985, 4, 43.

4. Silva, J. A.; Dissertação de Mestrado, Universidade Federal da Paraíba, Brasil, 1999.

5. Cesar, M. A. A.; Delgado, A. A.; Camargo, A. P.; Bissoli, B. M. A.; Silva, F. C.; STAB 1987, 5, 32 .

6. Ribeiro, F. J.; Lopes, J. J. C.; Ferrari, S. E.; Brasil Açucareiro 1987, 105, 26.

7. Vasconcelos, J. N.; Brasil Açucareiro 1987, 105, 41.

8. Barros Neto, B.; Scarminio, I. S.; Bruns, R. E.; Como Fazer Experimentos, Ed. da Unicamp: Campinas, 2001.

9. Pereira, A. C.; Kubota, L. T.; Quim. Nova 2004, 27, 725.

10. Pereira-Filho, E. R.; Poppi, R. J.; Arruda, M. A. Z.; Quim. Nova 2002, 25, 246.

11. Okolo, B.; Johnston, J. R.; Berry, D. R.; Biotechnol. Lett. 1987, 9, 431.

12. Amorim, H. V.; Resumos da Semana de Fermentação Alcoólica, Piracicaba, Brasil, 1985.

13. Cabezas, W. A. R. L.; Trivelin, P. C. O.; Silveira, J. A. G.; STAB 1990, 9 , 41.

14. Novaes, F. V.; Stupiello, J. P.; Oliveira, E. R.; Valsechi, O.; Resumos do I curso de extensão em tecnologia de aguardentes, Piracicaba, Brasil, 1974. 